(C)2007 IEEE. Personal use of this material is permitted. However, permission to reprint/republish this material for advertising or promotional purposes or for creating new collective works for resale or redistribution to servers or lists, or to reuse any copyrighted component of this work in other works must be obtained from the IEEE 


\title{
An Integrative view of the concept of Digital Ecosystem
}

\author{
Hai Dong, Farookh Khadeer Hussain, Elizabeth Chang \\ Digital Ecosystem and Business Intelligence Institute \\ Curtin University of Technology \\ Perth, Australia \\ Email: \{hai.dong, farookh.hussain, elizabeth.chang\}@cbs.curtin.edu.au
}

\begin{abstract}
In the literature we fid two broad definitions of the concept of Digital Ecosystem, hence leading to confusion and ambiguity with regards to its semantic interpretation. In this paper we make use of ontology, which is a well-known tool for knowledge sharing, in order to present an integrative view of the concept of Digital Ecosystem. We implement the ontology by using Protégé-owl.
\end{abstract}

\section{Keywords-Digital Ecosystem; ontology; Protégé-owl}

\section{INTRODUCTION}

The appearance of Digital Ecosystems is a result of the natural existence of Business Ecosystem, along with the evolution of business network and information technology. The goal of Digital Ecosystems is to improve the efficiency of the communication between internal agents and to formally structure the existing Business Ecosystem.

In the literature there are two broad definitions of the concept of Digital Ecosystems. Soluta.net propose a definition from a structural and functional perspective, which sees Digital Ecosystem as an open-source network environment for business mainly including small and medium enterprises to interact with others in an effective and efficient way [3]. On the contrary, the definition provided by Digital Ecosystem and Business Intelligence (DEBI) Institute sees it as "an open, loosely coupled, domain clustered, demand-driven, selforganizing and agent-based environment in which each species is proactive and responsive for its own benefit and profit" [2] [4].

As a result of these definitions, there is confusion regarding the concept and semantic interpretation of Digital Ecosystems. To solve this conceptual ambiguity and help researchers better understand we intend to develop a conceptual model of Digital Ecosystems. The conceptual model is built by using ontological notations.

\section{Notation System For OntOlogy RePRESENTATION}

Before we represent the ontological model for digital ecosystem, a notation system for representing ontology is introduced in this section. The notation system utilized in the ontological representation is based on Chang et al.'s work, which consists of four basic notations as Table 1[1]. Although in the past we usually used to employ UML (Unified Modeling Language) to represent an ontology model, due to its complex symbols categories, UML cannot efficiently help people better understand the shared knowledge. This notation system simplifies the symbols and its symbols are closer to the principle of ontology which is the combination of shared concepts and relationships between concepts [5].

TABLE I. ONTOLOGY NOTATION SYSTEM

\begin{tabular}{|l|l|}
\hline Ontology Notation & \multicolumn{1}{|c|}{ Semantics of the Notation } \\
\hline & $\begin{array}{l}\text { Double-field Box represents the Ontological } \\
\text { Concepts. }\end{array}$ \\
\hline- relation $^{-}-$ & $\begin{array}{l}\text { A dotted line represents Ontology Concept } \\
\text { Association Relation which represents a } \\
\text { Concept is closely related to another concept. } \\
\text { The relationship name can be noted above the } \\
\text { dotted line. }\end{array}$ \\
\hline$\rightarrow$ & $\begin{array}{l}\text { Open-arrow line represents Composition and } \\
\text { Aggregation or Part-of relationship between } \\
\text { Upper Ontology Concept and Lower Ontology } \\
\text { Concept. }\end{array}$ \\
\hline & $\begin{array}{l}\text { Solid-arrow line represents Generalization and } \\
\text { Specification relation, which is a relation } \\
\text { between Upper Generic Concept and Lower } \\
\text { Specific Concept. }\end{array}$ \\
\hline
\end{tabular}

III. Digital ECOSYSTEM ONTOLOGY

\section{A. Hierarchy of Digital Ecosystem Ontology Concepts}

Broadly speaking, digital ecosystem consists of two basic parts or components - species and an ecosystem environment. Each species can be viewed as an individual or an organization and has its own role to play. The species work together to take care of their living environment; on the other hand, environment supports species' survival and development. There are three basic types of species - biological species, economic species and digital species. Thus, the hierarchy of digital ecosystem concepts is shown in Figure 1, below using the above introduced notation system.

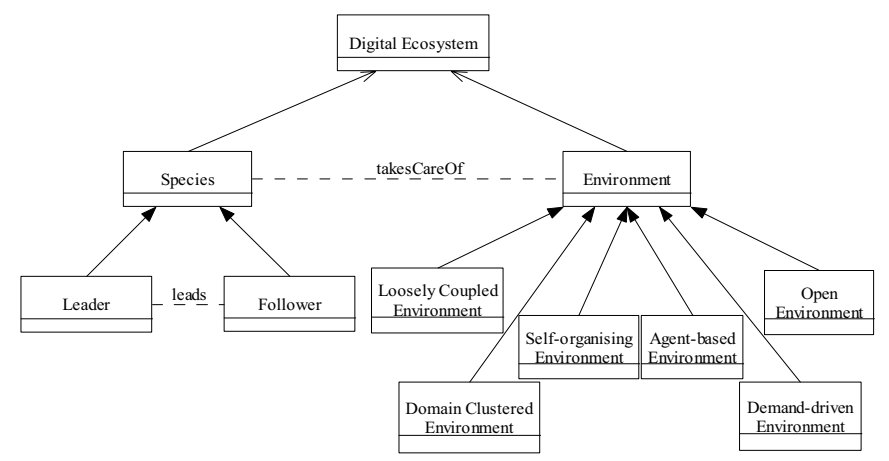

Figure 1. Digital ecosystem concepts hierarchy. 


\section{B. Species Ontology}

We regard Specie as a basic element with in Digital Ecosystem. Specie(s) come from a certain domain, play dual roles (role of a server in which it provides available services to other species and role of a client, who request service), follows the rules (norms or regulations) of digital ecosystem, is proactive for or is responsive for own profit or benefit and carries out tasks that relates to Profit or Benefit.

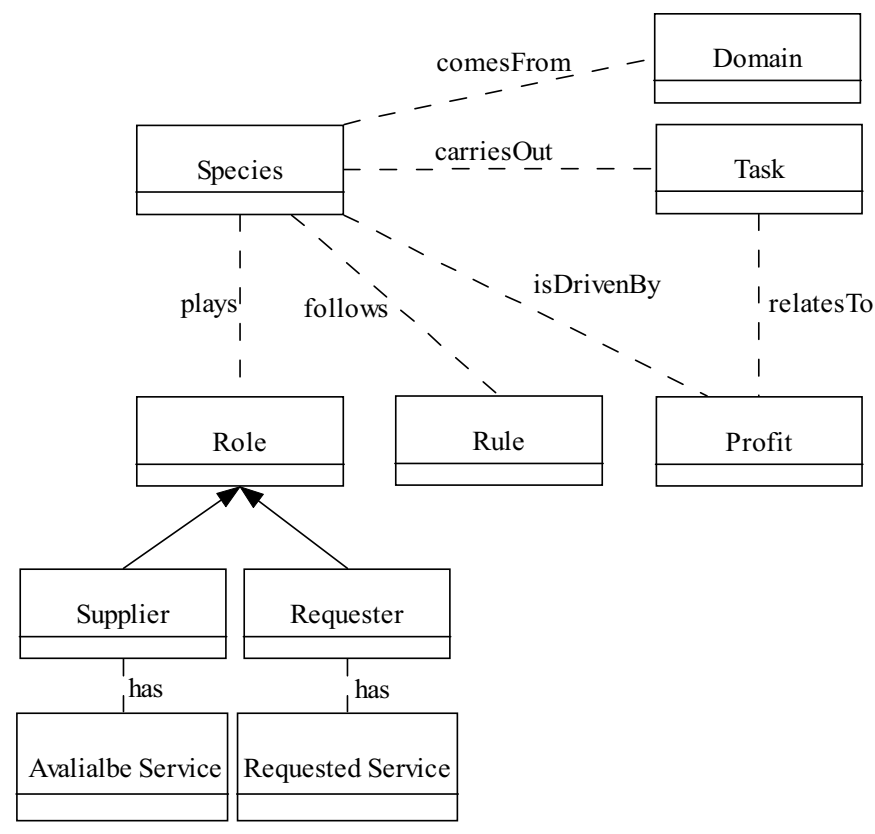

Figure 2. Species Ontology.

We present the Species Ontology as the combination of ontology name and a tuple where the elements of the tuple can be complex elements as defined below:

Species [Species, Domain, Task, Profit, Rule, Role, Supplier, Requester, Available Service and Requested Service] where

'Species' refers to the definitions above.

'Domain' refers to a field in digital ecosystem where a species has common interest with other species.

Task' refers to a mission that a species is executing, to survive and achieve its goals in the environment of digital ecosystem.

'Profit' or 'Benefit' means a social or economical advantage that a species gains by digital ecosystem.

'Rule' refers to a common or special regulation that a species should follow in the environment of digital ecosystem.

'Role' refers to the role of a specie in the interaction with other specie(s). There are two major roles in digital ecosystem interactions - server and client. In contrast to the other network architectures, in which each agent has a fixed role, specie(s) in digital ecosystem are able to play the dual roles at the same time.
'Supplier' is a role played by a specie that provides a given service to other species.

'Requester' is a role played by a specie that requests a given service from other species

'Available Service' is a service that a supplier provides.

'Requested Service' is a service that a requester needs.

\section{Leader Ontology and Follower Ontology}

Before we define the concept of a leader, in the context of Digital Ecosystems and the concept of a follower, in the context of Digital Ecosystems, the organizational structure of species needs to be discussed. Since the prime characteristics of Digital Ecosystem are self-organization and collaboration, species in Digital Ecosystems form a hierarchical organization, of flexible structures. Here a concept - swarm comes into picture. A swarm as defined by Chang ad West el al is 'a set of species which have common characteristic and is able to interact and engage directly or indirectly with each other' [4]. With swarm intelligence technology, the species that have common interest(s) may group and cooperate, to share a problem and to collaboratively carry out a task.

In a swarm, a leader is chosen from species, to direct and facilitate the swarm, to represent the swarm to interact with other swarms [4]. Besides these functions, the leading species has same features as other species.

The subclasses of Species Concept - Leader Concept and Follower Concept inherit all properties from Species Concept. The only difference is the relationship between Leader and Follower; a leader guides all followers, while all the followers follow a leader in a swarm.

The ontological representation of the concept of leader and follower is shown in Fig. 3 and Fig. 4 respectively. 


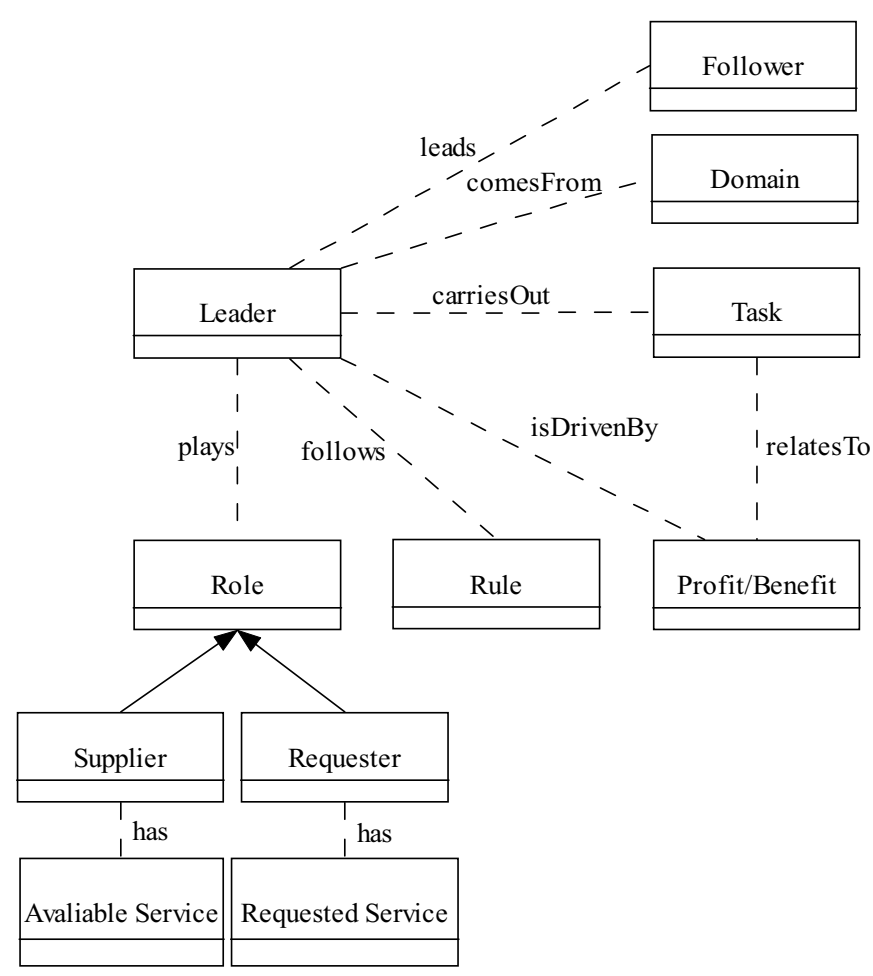

Figure 3. Leader Ontology.

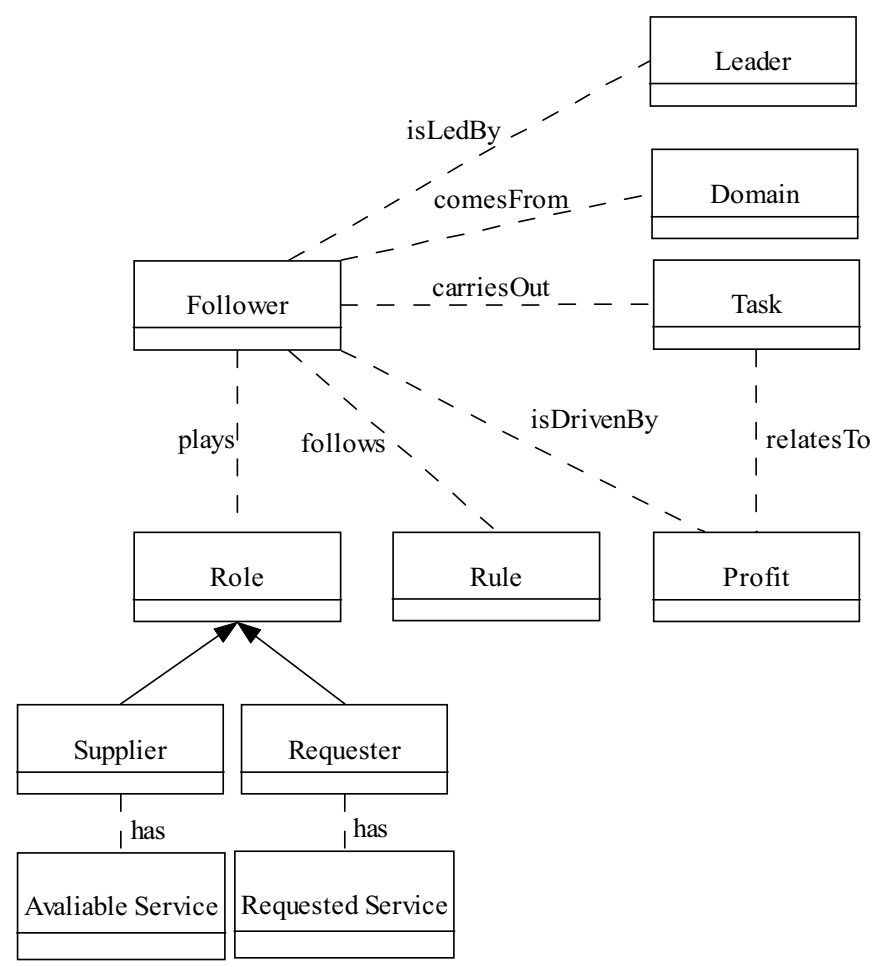

Figure 4. Follower Ontology.

\section{Environment Ontology}

In the context of Digital Ecosystem, environment can be defined as the conceptualization of environment that is supported by technologies, provided by species and additionally supports services to the constituent species. The environment has six characteristic features, namely - Open, Loosely Coupled, Demand-driven, Domain Clustered, Selforganizing and Agent-based Environment. The Environment Ontology is shown in Fig. 5 through the use of notation system.

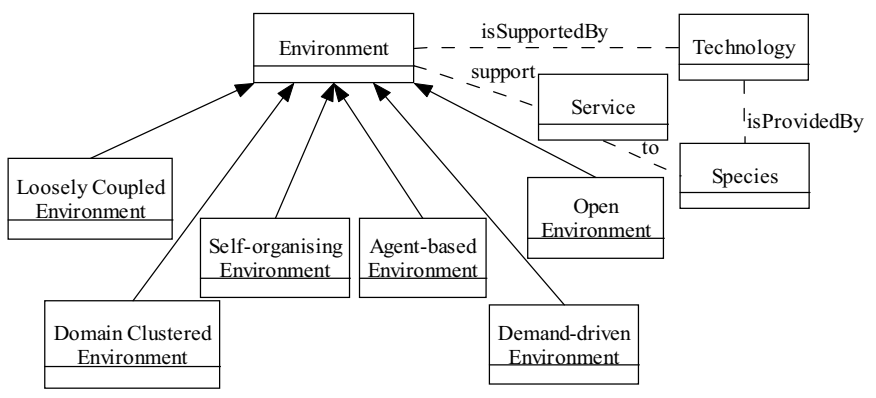

Figure 5. Environment Ontology.

We present the Environment Ontology as the combination of ontology name and a tuple where the elements of the tuple can be complex elements as defined below:

Environment [Environment, Technology, Service, Species, Open Environment, Loosely Coupled Environment, Demanddriven Environment, Domain Clustered Environment, Selforganizing Environment and Agent-based Environment] where

'Environment' refers to definition above.

'Technology' will be defined in the next section.

'Open' refers to a transparent environment where all interactions are visible [4].

'Loosely Coupled' refers to a freely bound and open relationship between species in digital ecosystem [4].

'Demand-driven' means that species actively join a community on their own interest [4].

'Domain Clustered' refers to an environment which consists of the field where some species have common interest [4].

'Self-organizing' means that species are capable of acting autonomously, making decisions and carrying out tasks in digital ecosystem [4].

'Agent-based' refers to an environment which contains human individuals, information technologies and tools that facilitate interaction and knowledge sharing between agents along with the resources that help maintain synergy between he agents [4].

\section{Prototype}

To realize and implement our design, we built the conceptual model by means of Protégé-owl. The graphical view of Hierarchy of Digital Ecosystem Ontology concepts in Protégé-owl is shown in Fig. 6. 


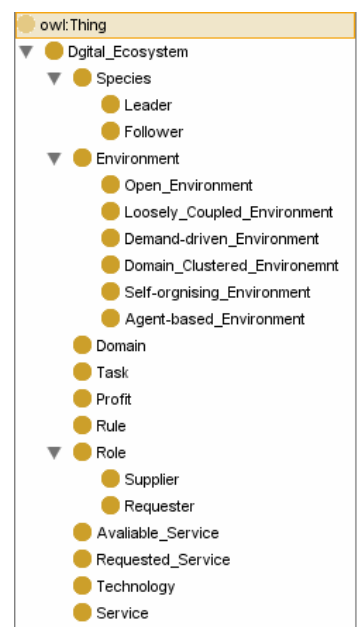

Figure 6. Hierarchy of Digital Ecosystem Ontology concepts in Protégé-owl.

\section{CONCLUSION AND FUtURE WORKS}

In this paper, we presented an integrative view of two distinct definitions of the concept of Digital Ecosystems. We use made use of ontology to represent the conceptual model of Digital Ecosystem and its subcomponents.
This paper proposes and explains the concept of Digital Ecosystem and its subclasses - Species and Environment. In addition, the subclasses of Species - Leader and Follower, and the subcomponents of Environment - six different characteristics of environments are explained through the use of the notation system introduced to substitute UML. Finally, we implement the prototype in Protégé-owl.

\section{REFERENCES}

[1] E. Chang, T. S. Dillon and F.K. Hussain, Trust and Reputation for Service Oriented Environments-Technologies for Building Business Intelligence and Consumer Confidence, London: John Wiley \& Sons, 2005, pp.131-153.

[2] E. Chang, M. Quaddus and R. Ramaseshan, "The vision of DEBI Institute: digital ecosystems and business intelligence," DEBII, 2006.

[3] P. Ferronato, "Introduction to the digital business ecosystem project," presented at MDA Technical Forum, Tokyo, 2004.

[4] E. Chang and M. West, "Digital Ecosystem - A next generation of the collaborative environment," presented at iiWAS 2006, Yogyakarta, 2006.

[5] N. Guarino, "Ontology-driven conceptual modeling," presented at 21st International Conference on Conceptual Modelling, Tampere, 2002, p.10. 